\title{
Rapid Detection of Heavy Metals on Waste-Water Polluted Soils Using Laser Induced Breakdown Spectroscopy (LIBS)
}

\author{
Heri Sugito, Ali Khumaeni, Qidir Maulana Binu Soesanto \\ Department of Physics, Faculty of Science and Mathematics, Diponegoro University, Semarang, Indonesia, $\underline{\text { herinuha@gmail.com }}$
}

\section{A R T I C LE INFO}

\section{Article history:}

Received : 5 April 2020

Accepted : 27 May 2020

Available online : 10 June 2020

\section{Keywords:}

LIBS

Heavy metal

Polluted soil

\begin{abstract}
A B S T R A C T
The Laser Induced Breakdown Spectroscopy (LIBS) method was successfully used to detect heavy metal elements in the soil polluted by waste-water from paper mills. The study was conducted using a Nd: YAG pulse laser with a wavelength of $532 \mathrm{~nm}$ at $83 \mathrm{~mJ}$ energy and 5 torr air pressure. The laser is fired at a soil sample that has been made in the form of pellets to produce plasma. The plasma emission spectrum formed is then detected by multichannel analyzer (OMA) to obtain the emission line spectrum that represents the content of atoms and molecules in the soil sample. The spectrum detected by OMA is then compared to the standard reference spectrum at NIST (National Institute of Standards and Technology) to find out the contents of an element on a contaminated soil sample. several types of heavy metal elements $\mathrm{Fe}, \mathrm{Cr}, \mathrm{C}, \mathrm{Ca}$ and $\mathrm{Mn}$ in soils contaminated by wastewater were detected using LIBS method. Based on research results (figure 2), the LIBS method is very wellused for the detection of heavy metal content in polluted soils.
\end{abstract}

\section{Introduction}

The development of industrial estates in agricultural and surrounding areas causes a reduction in the area of agricultural land, pollution of soil and water bodies which can reduce the quality and quantity of agricultural products, disruption of the comfort and health of humans or other living things. In some waste-water in the paper industry, $\mathrm{Pb}$ (lead) is sometimes found which is dangerous for the environment. In addition to $\mathrm{Pb}$, soils contaminated by paper mill waste-wateralso contain other heavy metal elements such as $\mathrm{Zn}$ (zinc), Cd (cadmium), Cr (chromium), $\mathrm{Cu}$ (copper), Fe (iron), Al (aluminum) and $\mathrm{Hg}$ (mercury).

The laser-induced spectroscopy (LIBS) method has been widely applied in the detection of heavy metals in polluted soils. Gibaek Kim et.al [1], utilize the LIBS method by adding a chemometric method to identify the content of heavy metals in contaminated soil. From the research conducted by Akhtar et.al [2], successfully combined an external magnetic field $(0.3 \mathrm{~T})$ with the LIBS method for detection of $\mathrm{Cr}$ in soil samples. The LIBS method has also been successfully applied to detect heavy metals in soils such as $\mathrm{Cu}, \mathrm{Ni}, \mathrm{Cr}, \mathrm{Pb}, \mathrm{Fe}, \mathrm{Mn}, \mathrm{Ti}, \mathrm{Si}, \mathrm{Al}$ [3-5].

Various methods besides LIBS have also been carried out to detect the content of heavy metals in polluted soils. Several analytical techniques are used to identify the element content in the soil to determine the level of environmental pollution [6].

Atomic absorption spectroscopy (AAS) technique is used for the analysis of heavy metal contamination in the soil around gold mining exploration in the city of Palu[7]. But these conventional methods require complex and difficult sample preparation in the laboratory. In addition, the XRD and AAS methods require a very long time to analyze pollution on soils with a very large area so that the method is not effective and efficient. Therefore, it is necessary to develop a method that can be used for analysis of soil pollution with fast time, does not require complicated sample preparation, is able to detect all elements contained in the target sample simultaneously and has a high level of sensitivity for identification of impurities and elements Minor which is dangerous to the soil.

Soil samples are samples that have characteristics that are difficult to analyze using the LIBS method because of the matrix effect in the form of physical and chemical properties. However, this methode have succeeded in analyzing heavy metal pollution in soils using LIBS method [8]. As a result, toxic and heavy metal impurities in the soil can be identified with a high level of sensitivity (ppm order). These results promise the possibility of using the LIBS method for elemental analysis in polluted soils.

Lidiane et al. (2009) conducted an experiment on LIBS for the determination of cadmium in soil [5]. The soil sample was prepared in the form of pellet prior to analysis. They concluded that the LIBS could be employed to a screening of cadmium pollution in soil. Pandhija et al. demonstrated an experiment on LIBS for the determination of heavy metals $\mathrm{Pb}$ in soil 
[6]. Semi-quantitative analysis of $\mathrm{Pb}$ in the soil can be realized with a detection limit of $45 \mathrm{ppm}$. Various studies on soil analysis by using LIBS have been made as reported elsewhere [5, 7-11]. There is a lack of information dealing with the detection of oil pollution in soil by using LIBS.

Recently, laser-induced breakdown spectroscopy (LIBS) has become a great method for qualitative and quantitative analyses of sample target in various kinds of samples including liquid , metals $[12,13]$ and solid [14]. In this technique, a pulse neodymium yttrium aluminum garnet (Nd: YAG) laser is focused on/in a sample to induce a luminous plasma. In the plasma region, molecules, and atoms ablated from the sample can be effectively dissociated and excited. Compared to other conventional analytical methods, standard LIBS is much superior because the rapid analysis can be performed without tedious sample preparation, and it has low-cost experimental equipment [15]. Several studies have reported on the application of standard LIBS for the analysis of soil target [7]. Del'Agglio et al. (2011) have detected heavy metals elements such as $\mathrm{Cr}, \mathrm{Cu}, \mathrm{Pb}$, and $\mathrm{Zn}$ in soil. A comparative study has been made by using ICP-OES. The correlation between ICP-OES and LIBS was confirmed by a satisfactory agreement [16]. However, in standard LIBS method using pulse Nd: YAG laser, the soil sample should be prepared in the form of pellet for effective dissociation and excitation process.

This research examines the LIBS method for identification of $\mathrm{Fe}, \mathrm{Cr}, \mathrm{Mn}, \mathrm{Ca}, \mathrm{C}$ elements and analysis of the potential for toxic metal contents in soils polluted by the paper industry waste.

\section{Methodology}

The equipment used in this study is (1) Nd: YAG laser as an energy source (1064 NM, $150 \mathrm{~mJ}, 7 \mathrm{~ns}$ ), (2) Optical Multichannel Analyzer (OMA) connected to (3) optical fibers, (4) Chamber as a sample container connected to (5) vacuum pumps and (6) pressure measuring equipment, (7) mirrors, (8) lenses for laser beam focusing and (9) computer Each sample is given the same experimental treatment, which is raised at a low pressure of 5 Torr, $83 \mathrm{~mJ}$ energy. The arrangement of equipment used in the study is shown in the following fig. 1.

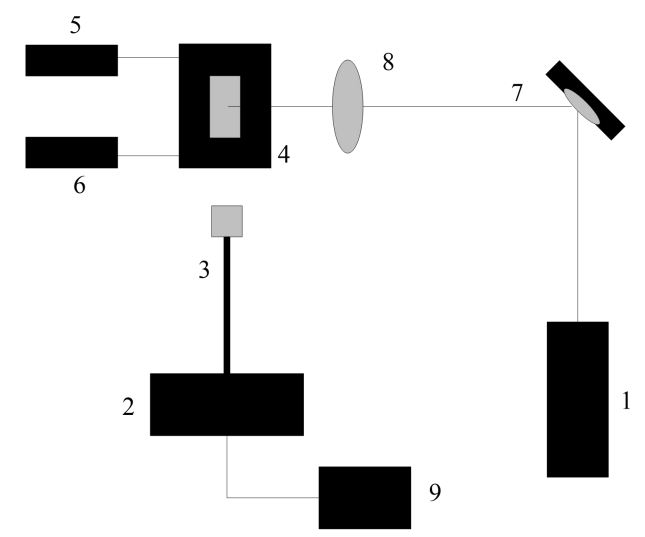

Fig. 1: The arrangement of LIBS tools in research
The samples used in this research were soil with contaminated waste and soil uncontaminated. Determination of an element in a sample can be known through spectrum graph data. The data for each detected wavelength intensity is matched with the Atomic Spectra Database Line Form reference data from NIST (National Institute of Standards and Technology) Physical Measurement Laboratory.

\section{Results and Discussions}

Plasma produced from the mechanism of generation by laser beam shooting has unique characteristics. That is because the ionized and excited gas from the material constituents emits specific elements in the form of wavelengths with laser color and plasma sizes that vary according to the energy level of the transition. Laser interaction with the target will cause an increase in temperature on its surface. This will cause ablation on the target surface. The target will absorb the energy of the laser beam so that the atoms at the base energy level will transition to higher energy levels. The accumulated energy will become vapor and cause the bonding of the material atoms will be broken. This process will produce plasma emissions in accordance with the conditions of the given experiment.

In this research, the laser plasma spectroscopy method is used to detect the content of heavy metal elements in uncontaminated soil samples (fig. 2a) and polluted soils (fig. $2 \mathrm{~b}$ and $2 \mathrm{c}$ ). The resulting spectrum is captured by the spectrum analyzer (OMA), then compared to the standard data spectrum at NIST. The obtained spectrum is limited to wavelengths of $420 \mathrm{~nm}-435 \mathrm{~nm}$. With this LIBS method, obtained spectrum data from each sample as shown in figures $\mathrm{a}, \mathrm{b}$, and c.

Fig. 2a shows the spectrum of the detection results in a non-polluted soil sample (as sample 1). Sample 1 is paddy soil taken at a distance of $100 \mathrm{~m}$ outside the central area of sewage treatment and paper waste water spills. From the spectrum obtained, the detected elements of Ca I $(422.67 \mathrm{~nm})$, Fe I (427.17nm), Mn I (430.56nm and 430.82nm) and C III $(432.56 \mathrm{~nm})$. The Ca I element in uncontaminated soil samples has the highest intensity, which is around 6321, then Mn I with an intensity of 1300, C III with an intensity of 510, Fe I with an intensity of 221. Ca is a nutrient produced from weathering primary minerals such as fiedstar, ferrous magnesian, volcanic glass, mica, zeolite and apatite in the soil.

Fig. $2 \mathrm{~b}$ shows the spectrum of detection results in soil samples 2, ie soil samples taken at a distance of 1 meter from the waste treatment spill. The graph shows elements of Fe I (421.54nm), Cr I (425.43 nm and $427.49 \mathrm{~nm}), \mathrm{Ca} I(428.30 \mathrm{~nm}$ and $428.95 \mathrm{~nm}), \mathrm{C}$ II $(431.85 \mathrm{~nm})$. From the graph shows the heavy metal element $\mathrm{Cr}$ which is a product of waste. Fig. 2c also shows the presence of $\mathrm{Cr}$ element in soil sample 3 , ie soil samples taken in the community 30 meters from the waste center. However, the intensity of $\mathrm{Cr}$ element in the soil sample 2 is higher, namely 710 and 1803, while the sample 3 has the intensity of 433 and 539. This shows that the soil sample 2 has a greater $\mathrm{Cr}$ element content than the soil of sample 3. 

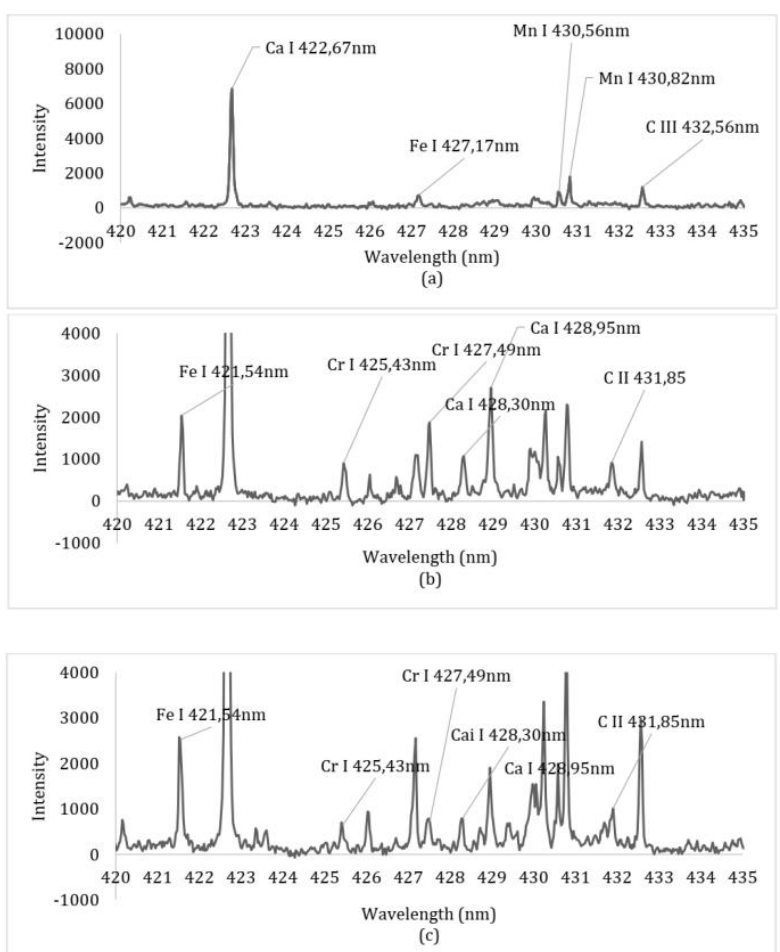

Fig. 2: The spectrum of (a) uncontaminated soils (sample 1), (b) polluted soil of sample 2 (soil samples taken at a distance of 1 meter from the waste treatment spill), (c) polluted soil of sample 3 (soil samples taken in the community 30 meters from the waste center), each spectrum are in the wavelength range of $420 \mathrm{~nm}-435 \mathrm{~nm}$

Spectrum characteristics that represent elemental content have varying intensities. Each element has a different energy level from each other. The intensity of the spectrum resulting from the spectroscopic method is influenced by three factors, namely transition probability, population at each level, and sample concentration. The higher the intensity of the spectrum of an atom, it can be concluded the greater the concentration of atoms in the sample.

\section{Conclusions}

The spectrum confirms that the LIBS method is able to detect heavy metal elements in contaminated soil. The LIBS method clearly detects the presence of elements $\mathrm{Fe}, \mathrm{Cr}, \mathrm{Mn}, \mathrm{Ca}, \mathrm{C}$ with varying wavelengths which are waste paper products that pollute the soil.

\section{Acknowledgment}

The research was supported by Development Research and Application Diponegoro University.

\section{References}

1. G Kim, Y-J Yoon, H-a Kim, H-J Cho and K Park, J. Spectrochimica Acta Part B: Atomic Spectroscopy 134 17-24 (2017)

2. M. Akhtar, A. Jabbar, S. Mehmood, N. Ahmed, R. Ahmed, M. A. Baig, J. Spectrochimica Acta Part B: Atomic Spectroscopy 148 143-151 (2018)

3. Mirdat, Patadungan Y. S., and Isrun, J. Agrotekbis 12 127-134 (2013)

4. A. Khumaeni, M Ramli, Y Deguchi, Y I Lee, N Idris, K H Kurniawan, T J Lie, and K Kagawa, Applied Spectroscopy 6212 1344-1348 (2008)

5. D. S. Jr. Lidiane C. Nunes, L.C. Trevizan, Q. Godoi, F. O. Leme, J. W. B. Braga, and F. J. Krug, Spectrochim. Acta Part B 641073 (2009)

6. A. M. Popov, F. Colao, and R. Fantoni, J. Anal. At. Spectrom. 25837 (2010)

7. V. K. Unnikrishnan, R. Nayak, K. Aithal, V. B. Kartha, C. Santhosh, G. P. Guptac, and B. M. Suric, Anal. Methods 51294 (2013)

8. M. V. Belkov, V. S. Burakov, A. De Giacomo, V. V. Kiris, S. N. Raikov, and N. V. Tarasenko, Spectrochim. Acta Part B 64899 (2009)

9. K. Rifai, S. Laville, F. Vidal, M. Sabsabi, and M. Chaker, J. Anal. At. Spectrom. 27, 276-283 (2012)

10. E. M. Cahoon and J. R. Almirall, Anal. Chem. 84 2239-2244 (2012)

11. C. M. Li, Z. M. Zou, X. Y. Yang, Z. Q. Hao, L. B. Guo, X. Y. Li, Y. F. Lu, and X. Y. Zeng, J. Anal. At. Spectrom. 29 1432-1437 (2014)

12. P. Yaroshchyk, D. L. Death, and S. J. Spencer, J. Anal. At. Spectrom. 27 92-98 (2012)

13. A. K. Myakalwar, S. Sreedhar, I. Barman, N. C. Dingari, S. V. Rao, P. P. Kiran, S. P. Tewari, and G. M. Kumar, Talanta 87 53-59 (2011)

14. E. M. Cahoon and J. R. Almirall, Appl. Opt. 49 C49-C57 (2010)

15. D. A. Cremers, and L. J. Radziemski, "Handbook of Laser-Induced Breakdown Spectroscopy John Wiley and Sons," Ltd, Chichester (2006)

16. A. W. Miziolek, V. Palleschi, and I. Schechter, "Laser-induced breakdown spectroscopy (LIBS): Fundamentals and Applications," Cambridge University Press (2006) 